\title{
A NOTE ON SPUN KNOTS
}

\author{
C. MCA. GORDON ${ }^{1}$
}

\begin{abstract}
This paper contains some remarks about spinning which show, in particular, that the spun reef knot is equivalent to the spun granny.
\end{abstract}

I am grateful to J. J. Andrews for having some time ago drawn my attention to the following problem concerning spun knots: Is the spun reef equivalent to the spun granny? Since it seems that several knot theorists have at one time or another been interested in this problem, it may be worth recording the following simple solution.

Spinning was introduced by Artin [2], and the more general process of $p$-spinning seems to have been used first by Epstein [4]. Since then it has been studied and used by many other authors; see [1], [3], [5], [7], etc. The definition (which is valid in DIFF or PL) is probably best expressed as follows.

Let $K=(M, N)$ be a pair consisting of a closed, oriented, manifold $M$ of dimension $m$, and a closed, oriented (locally flat) submanifold $N$ of dimension $n$. Let $\bar{K}=(\bar{M}, \bar{N})$ be the pair obtained by removing the interior of a standard ball pair $\left(B^{m}, B^{n}\right) \subset(M, N)$. Then the $p$-spin of $K, p \geqslant 0$, is the oriented pair

$$
\sigma_{p}(K)=\partial\left(\bar{K} \times D^{p+1}\right),
$$

where $D^{p+1}$ is the $(p+1)$-disc with some fixed orientation.

In particular, if $K$ is a knot of $S^{n}$ in $S^{m}$, then $\sigma_{p}(K)$ is a knot of $S^{n+p}$ in $S^{m+p}$.

Let $\cong$ denote orientation-preserving isomorphism (of pairs).

It is well known that $p$-spinning behaves nicely with respect to connected sum:

LEMMA 1. $\sigma_{p}\left(K_{1} \# K_{2}\right) \cong \sigma_{p}\left(K_{1}\right) \# \sigma_{p}\left(K_{2}\right)$.

Proof. Using \# to denote boundary connected sum, we have

$$
\begin{aligned}
\sigma_{p}\left(K_{1} \# K_{2}\right)=\partial\left(\left(\overline{K_{1} \# K_{2}}\right) \times D^{p+1}\right) \\
\cong \partial\left(\left(\bar{K}_{1} \dot{K_{2}}\right) \times D^{p+1}\right) \cong \partial\left(\left(\bar{K}_{1} \times D^{p+1}\right) \#\left(\bar{K}_{2} \times D^{p+1}\right)\right) \\
\cong \partial\left(\bar{K}_{1} \times D^{p+1}\right) \# \partial\left(\bar{K}_{2} \times D^{p+1}\right)=\sigma_{p}\left(K_{1}\right) \# \sigma_{p}\left(K_{2}\right) .
\end{aligned}
$$

Received by the editors December 19, 1974.

AMS (MOS) subject classifications (1970). Primary 55A25, 57C45.

Key words and phrases. Knots, spinning, p-spinning.

${ }^{1}$ Supported by a Science Research Council Postdoctoral research Fellowship. 
Reversing the orientation of the pair $K$ gives

LeMma 2. $\sigma_{p}(-K) \cong-\sigma_{p}(K)$.

Now observe that $D^{p+1}$ has an orientation-reversing automorphism, $r$, say. Then id $\times r: \bar{K} \times D^{p+1} \rightarrow \bar{K} \times D^{p+1}$ is orientation-reversing, and hence, restricting to the boundary, we get

LeMma 3. $\sigma_{p}(K) \cong-\sigma_{p}(K)$.

Note. For $p=0$, this is just the statement $K \#-K \cong-(K \#-K)$.

Lemmas 1,2 , and 3 clearly imply, in particular,

Corollary 4. $\sigma_{p}(K \#-K) \cong \sigma_{p}(K \# K)$.

Taking $K$ to be the trefoil knot and $p=1$ now gives an affirmative answer to the question raised at the beginning.

REMARK. As the referee has kindly pointed out, interest in the problem is due to the fact that the reef and granny themselves are known to be inequivalent [6]. Hence the above may be interpreted as saying that, as a homomorphism on the graded semigroup of knots, the operation of $p$ spinning is not injective.

ADDED IN PROOF. A different proof that the spun reef and spun granny are equivalent has been given by Dennis Roseman (preprint, University of Iowa).

\section{REFERENCES}

1. J. J. Andrews and D. W. Sumners, On higher-dimensional fibered knots, Trans. Amer. Math. Soc. 153 (1971), 415-426. MR 42 \#6808.

2. E. Artin, Zur Isotopie zweidimensionaler Flächen in $R_{4}$, Abh. Math. Sem. Univ. Hamburg 4 (1926), 174-177.

3. S. Cappell, Superspinning and knot complements, Topology of Manifolds (Proc. Inst. Univ. of Georgia, Athens, Ga., 1969), Markham, Chicago, Ill., 1970, pp. 358-383. MR 43 \#2711.

4. D. B. A. Epstein, Linking spheres, Proc. Cambridge Philos. Soc. 56 (1960), 215-219. MR 22 \#8514.

5. C. McA. Gordon, Some higher-dimensional knots with the same homotopy groups, Quart J. Math. Oxford Ser (2) 24 (1973), 411-422. MR 48 \# 5089.

6. H. Seifert, Verschlingungsinvarianten, S.-B. Preuss. Akad. Wiss. 26 (1933), 811-823.

7. D. W. L. Sumners, On an unlinking theorem, Proc. Cambridge Philos. Soc. 71 (1972), 1-4. MR 44 \#7536.

DPMMS, 16 Mill Lane, Cambridge, CB2 1SB, England 